\title{
A PROGAMMED CONSTRUCTION FOR THE POSITIONAL CONTROL
}

\author{
V.D. Batuhtin \\ Institute of Mathematics and Mechanics, Ural \\ Scientific Center of the Academy of Scienes \\ of the USSR. \\ S. Kovalevskaja st. 16, Sverdlovsk, USSR.
}

Let the motion of a competitively controlled system be described by the differential equation

$$
d x / d t=f(t, x, u, v), \quad x\left(t_{0}\right)=x_{0},
$$

where $x \in R^{n} \quad$ is the phase vector of the system; $u$ and $v$ are the vectors controlling the actions of the players with restrictions $u[t] \in P \subset R^{P}, v[t] \in Q \subset R^{q} ; P$ and $Q$ are compacts; the function $f(t, x, u, v)$ is continuous in the totality of the arguments and continuously differentiable in $x$. In addition, we will assune that the formulated in [1] condition of uniform extendability of the solutions for the equation $(1)$ is fulfilled. Given are some instant $V_{0}>t_{0}$, a closed set $T \subset\left[t_{0}, V_{0}\right]$ a compact $\mathcal{U}=\left\{\left(V_{,}, m\right): V \in T, m \in \mathcal{U}_{V}\right\}$ in $R^{k+4}$, where $\mathcal{M}_{\mathfrak{V}}=\{m:(\vartheta, m) \in \mathcal{U}\}$ and a function $\omega(\vartheta, x, m)$ defined on the set $\left\{(\mathfrak{V}, x, m):(V, m) \in \mathcal{M}, x \in R^{n}\right\}$ is continuous in the totality of the arguments and continuously differentiable in $x$ in the domain $\omega_{0}<\omega<\omega^{\circ}$.

We will call a mixed strategy $\tilde{U} \div \mu_{\{t, x\}}(d u)$ of the first player a function $\mu_{\{t, x\}}$ (du) which puts the Borel regular normed measures $\mu(d u)$ on $P$ in correspondence to any position $\{t, x\}$. Let us define a motion $x\left[t ; t_{0}, x_{0}, \widetilde{U}\right]$ generated by the strategy $\tilde{L}$ as any uniform limit of Euler splines $\widetilde{x}_{\Delta}^{(k)}[t]$, for almost all $t \in\left[\tau_{i}^{(k)}, \tau_{i+1}^{(k)}\right)$ satisfying the equation

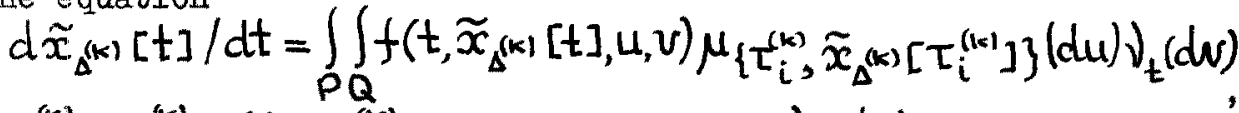

$$
\begin{aligned}
& \tau_{i+1}^{(k)}-\tau_{i}^{(k)} \leqslant \Delta^{(k)}, \quad \Delta^{(k)} \rightarrow 0 \text { for } k \rightarrow \infty, \nu_{t}(d v) \text { is a Borel }
\end{aligned}
$$


regular weak measurable in $t$ on $\left[t_{0}, V_{0}\right]$ function normed on $Q$, that is, a function

$$
\alpha(t)=\int_{Q} \varphi(v) \nu_{t}(d v)
$$

is a Lebesgue measurable function on $\left[t_{0}, V_{0}\right)$ for any arbitrary continuous function $\varphi(v) \in C(Q)$. Analogously a mixed strategy $\tilde{V} \div \nu_{\{t, x\}}(d v)$ of the second player and a motion generated by this strategy and also a motion generated by the couple $\{\tilde{U}, \widetilde{V}\} \quad$ are defined.

Problem I. For a fixed position $\left\{t_{0}, x_{0}\right\}$ and a number $C$ it is required to find a mixed strategy $\widetilde{U}$ which guarantees the inequality

$$
\min _{\mathcal{V} \in T} \min _{\mathfrak{j} \in \mathcal{M l}_{V}} \omega(\mathrm{J}, x[\mathrm{~J}], m) \leq c
$$

for any motion $x\left[t_{;} t_{0}, x_{0}, \widetilde{U}\right]$
Problem II. For a fixed position f $_{\left.0, x_{0}\right\}}$ and a number $C$ it is required to find a mixed strategy $\widetilde{V}$ which guarantees the inequality

$$
\min _{v \in T} \min _{j \in \mathcal{M}} \omega(\vartheta, x[q], m) \geqslant c
$$

for any motion $x\left[t ; t_{0}, x_{0}, \tilde{V}\right]$.

Let us introduce an auxiliary programmed construction for solving these problems. Namely on the space of generalized programmed controls - Borel regular measures $\eta_{t}=\eta_{t}$ (du,dv) defined and normed on $P \times Q$ for all $t \in\left[t_{0}, V_{0}\right)$ and weak measurable in $t$ on $\left[t_{0}, V_{0}\right)$ we will assign a totality of sets called programs.

Then let us define an elementary program $\left\{\mu_{t} \times \nu_{t}^{*}\left[t_{*}, \mathbb{V}\right)\right\}$ on $\left[t_{*}, \vartheta\right)$ as a set of all controls $\eta_{t}$ on $\left[t_{*}, \frac{7}{7}\right.$ which are represented in the form of the direct product $\eta_{t}=\mu_{t} \times \gamma_{t}^{*}$, where $\mu_{t} \in\left\{\mu_{t}\right\}$ and $J_{t}^{*}$ are weak measurable on $\left[t_{*}, \vartheta\right)$ Borel regular measures for any $t \in\left[t_{*}, \mathcal{V}\right)$ defined and normed on $P$ and $Q$ respectively.

We will put in correspondence to each position $\left\{t_{*}, x_{*}\right\}$

$\left(t_{x} \in\left[t_{0}, \bar{V}_{0}\right]\right)$ the quantity

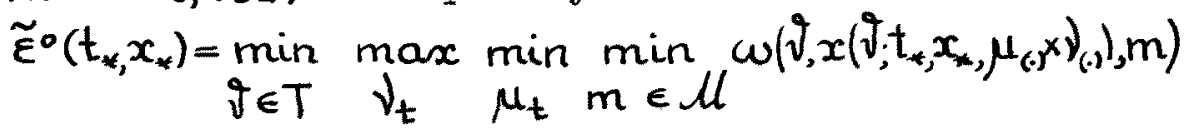

where $x(t)=x\left(t ; t_{*}, x_{*}, \mu_{(-)} \times \nu_{(-)}\right)$is a programmed motion satis- 
fying for almost all $t \in\left[t_{*}, \Im\right)$ the equation

$$
d x / d t=\iint_{P Q} f(t, x, u, v) \mu_{t}(d u) \nu_{t}(d v)
$$

Given by(2) optimal programmed controls $\mu_{t}^{0}, \nu_{t}^{0}$ and $m^{\circ} \epsilon l l$, $\vartheta^{\circ} \in T$ exist on account of weak compactness in themselves of the

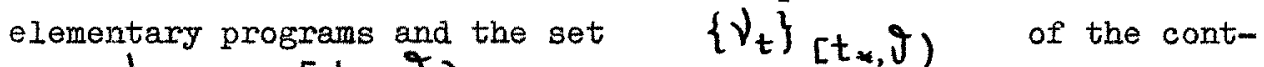
rols $V_{t}$ on $\left[t_{*}, \tau\right)$. Incidentally under the weak convergence of the sequences we understand the convergence in $*$ - weak topology of the sequences of continuous linear functionals defined by the Borel regular measures $\mu^{(k)}=\mu_{t}^{(k)} \cdot m(d t)$ $=v_{t}^{(k)} \cdot m(d t), m(\cdot)$ - the Lebesgue measure on ' $R^{\prime}$ '. We will say that the elementary program $\left\{\mu_{t} \times \nu_{t}^{\circ},\left[t_{*}, \dot{j}\right)\right\}$, where $j_{t}^{0}$ is an optimal control for $\left\{t_{*}, x_{*}\right\}$, is regular in position $\left\{t_{*}, x_{*}\right\}$, if the problem (2) has an essentially unique solution $\mu_{t}^{0}$ for the fixed control $\nu_{t}^{0}$, in addition, the minimal point $m^{\circ} \in M$ is also unique.

There is valid the following assertion which is an analogy of the maximum principle [2] in the case under study.

Theorem I. Let the regularity condition of the program

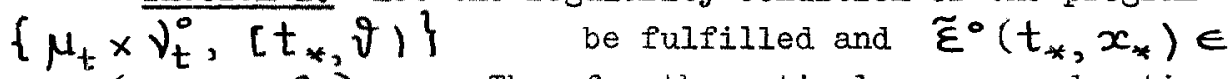
$\epsilon\left(\omega_{0}, \omega^{0}\right)$. Then for the optimal programmed motion $x^{\circ}(t)=x^{\prime}\left(t ; t_{*}, x_{*}, \mu_{(.)}^{0} \times \nu_{(,)}^{0}\right)$ there is the following minimax condition

$$
\begin{aligned}
& \iint_{P Q} j^{\prime}(\nu, t) f\left(t, x^{\circ}(t), u, v\right) \mu_{t}^{0}(d u) \nu_{t}^{0}(d v)= \\
= & \min _{\mu} \max _{\nu} \iint_{P Q} s^{\prime}(v, t) f\left(t, x^{0}(t), u, v\right) \mu(d u) \nu(d v)
\end{aligned}
$$

for almost all $t \in\left[t_{*}, \vartheta\right]$. Here $s^{\prime \prime}(J, t)=-[\partial \omega(V)$, $\left.\left.x^{\circ}(v), m^{0}\right) / \partial x\right]^{\prime} \cdot S\left(v, t_{*}, x^{0}(\cdot), \mu_{(\cdot)}^{0} \times \nu_{(\cdot)}^{0}\right), S\left(t, t_{*}, x(\cdot), \mu_{(\cdot)} \times \nu_{(.)}\right)$

is the fundamental solution matrix of the first variational approximation equation for equation (3) computed on the motion $x(t)=x(t$; $\left.t_{*}, x_{*}, \mu_{(-)} \times \nu_{(-)}\right)$, the prime denotes transposition. Let $\nu_{t}^{(k)} \underline{w}-\nu_{t}$ and $\left\{\eta_{t}\right\}^{*}$ be a set of all controls $\eta_{t}$ for each of which exists such a sequence $\left\{\mu_{t}^{(t-1)}\right\}$ 
that $\mu_{t}^{(k)} \times \nu_{t}^{(k)} \underline{w} \eta_{t}$. Then the weak closure of the set $\left\{\eta_{t}\right\}^{*}$ in $*$ - weak topology $C^{*}\left(\left[t_{*}, \vartheta\right] \times P \times Q\right)$ we will call the program $\Pi\left(\nu_{t}^{*}\right)$. We will say that the program $\Pi\left(\nu_{t}^{\circ}\right)$ is optimal, if a sequence which forms it is maximizing for $\left\{t_{*}, x_{*}\right\}$. We will call $\nu_{t}^{0}$ a regular control, if for any optimal $\Pi\left(\nu_{t}^{0}\right)$ the minimizing control $\eta_{t}^{0}$ in it is unique and also unique is $m^{*} \in \mathcal{M l}$. Let us denote by $\tilde{T}^{\circ}\left(t_{*}, x_{*}\right)$ the set of the problem's (2) solutions $g^{\circ}$ and by $\tilde{S}^{\circ}\left(t_{*}, x_{x} ; \mathcal{V}^{\circ}\right)$ the set of all vectors $\mathfrak{s}^{\circ}\left(\mathcal{V}^{\circ}, t\right), \boldsymbol{V}^{0} \in T^{\circ}\left(t_{*}, x_{*}\right)$ corresponding to all kinds of $\eta_{t}^{0}$ for $\left\{t_{*}, x_{*}\right\}$.

We shall suppose that for any $\left\{t_{*}, x_{*}\right\} \quad\left(t_{0} \leq t * \leqslant\right.$ $\left.\leqslant U_{0}\right), t_{*} \notin T^{0}\left(t_{*}, x_{*}\right)$, where $\widetilde{\varepsilon}^{0}\left(t_{*}, x_{*}\right) \in\left(\omega_{0}, \omega^{0}\right)$ and any Borel regular normed measure $\nu^{*}(d v)$ on $Q$ there exists an instant $\mathcal{J}^{\circ} \in T^{\circ}\left(t_{*}, x_{*}\right)$ for which two following conditions are fulfilled.

A. Any control $\nu_{t}^{0}$ is regular for $\left\{t_{*}, x_{*}\right\}$.

B. There exists a Borel regular normed measure $\dot{\mu}^{*}(\mathrm{du})$ on $P$ such that for any $\mathcal{J}^{\circ}\left(\mathcal{V}^{\circ}, t_{*}\right) \in \widetilde{S}^{\circ}\left(t_{*}, x_{*} ; g^{\circ}\right)$

$$
\iint_{P} s^{o}\left(v^{\circ}, t_{*}\right) f\left(t_{*}, x_{*}, u, v\right) \mu^{*}(d u) \nu^{*}(d v) \leqslant
$$

$$
\leqslant \min _{\mu} \max _{\nu} \int_{P Q} \int_{s^{\prime}}\left(v^{\circ}, t_{*}\right) f\left(t_{*}, x_{*}, u, v\right) \mu(d u) \nu(d v) .
$$

Theorem 2. Let $\tilde{\varepsilon}^{\circ}\left(t_{0}, x_{0}\right) \leq c \in\left(\omega_{0}, \omega^{0}\right)$ and conditions $A, B$ be fulfilled. Then the mixed strategy $\widetilde{U}^{(E)}$ which is
extremal [3] to the set $\tilde{W}_{c}=\left\{\{t, x\}: \widetilde{\varepsilon}^{\circ}(t, x) \leqslant C\right\}$ solves Problem I.

Let us denote by $\Sigma\left(t_{*}, x_{*} ; g^{0}\right)$ the set of all $\nu_{t}^{0}$ for $\left\{t_{*}, x_{*}\right\}$ and some $\mathcal{J}^{0} \in T^{0}\left(t_{*}, x_{*}\right), \quad \tilde{S}^{0}\left(t_{*}, x_{*}\right)=$

$$
=U_{T^{\circ}\left(t_{*}, x_{*}\right)} \tilde{S}^{\circ}\left(t_{*}, x_{*} ; \mathcal{V}^{\circ}\right) \text {. We shall now suppose that two follo- }
$$

wing conditions are fulfilled for ang $\left\{t_{*}, x_{*}\right\}: \tilde{E}^{\circ}\left(t_{*}, x_{*}\right) \in\left(\omega_{0}, \omega^{\circ}\right)$ instead of $A, B$.

C. Sets $\sum\left(t_{*}, x_{*} ; \mathcal{V}^{\circ}\right)$ are upper weak semicontinuous in each point $\mathfrak{J}^{0} \in T^{0}\left(t_{*}, x_{*}\right)$.

D. There exists $\nu_{*}\left(d^{*}\right)$ on $Q_{\text {f for any }} \mu_{*}(d u)$ on

$P$ such that for any $J^{\circ}\left(J^{\circ}, t_{*}\right) \in \widetilde{S}^{\circ}\left(t_{*}, x_{*}\right)$ 


$$
\begin{gathered}
\quad \iint_{P Q} s^{\prime}\left(v^{\circ}, t_{*}\right) f\left(t_{*}, x_{*}, u, v\right) \mu_{*}(d u) \nu_{*}(d v) \geqslant \\
\geqslant \max _{\nu} \min _{\mu} \int_{P} \int_{Q} s^{\prime}\left(\nu^{\circ}, t_{*}\right) f\left(t_{*}, x_{*}, u, v\right) \mu(d u) \nu(d v) .
\end{gathered}
$$

Theorem 3. Let $\tilde{\varepsilon}^{0}\left(t_{0}, x_{0}\right) \geqslant \mathcal{C} \in\left(\omega_{0}, \omega^{0}\right)$ conditions $C, D$ be fulfilled. Then the mixed strategy is extremal to the set $\widetilde{W}^{(c)}=\left\{\{t, x\}: \widetilde{\varepsilon}^{\circ}(t, x) \geqslant c\right\}$

and $\tilde{V}^{(e)}$ which solves Problem II.

With conditions A - D fulfilled simultaneously, the situation of equilibrium takes place.

If the saddle point condition $\min _{u \in P} \max _{v \in Q} s^{\prime} \cdot f(t, x, u, v)=\max _{v \in Q} \min _{u \in P} s^{\prime} \cdot f(t, x, u, v)$

for the minor game is fulfilled for any $s, t, x$, then the problems I, II are solvable in pure strategies.

$$
\text { References }
$$

1. V.D. Batuhtin, N.N. Krasovskil. The problem of programmed control on maximin, Izv. AN SSSR, Tehnicheskaja kibernetika, 1972, № 6 . ( Eussian ).

2. L.S. Pontrjagin, V.G. Boltjanskii, R.V. Gamkrelidze, E.F. Mistchenko. The mathematical theory of optimal processes, Fizmatgiz, Moscow, 1961. (Russian).

3. N.N. Krasovskii. The differential game of converging - evading, Izv. AN SSSR, Tehnicheskaja kibernetika, 1973, № 栏 2, 3. (Russian). 